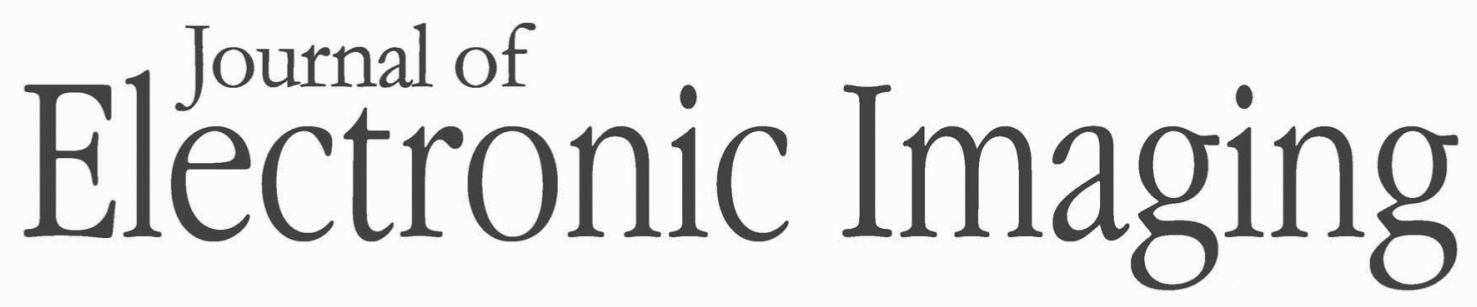

SPIEDigitalLibrary.org/jei

\title{
Special Section Guest Editorial: Video Surveillance and Transportation Imaging
}

Robert Loce Eli Saber

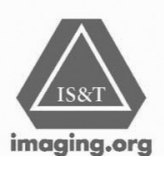




\title{
Special Section Guest Editorial: Video Surveillance and Transportation Imaging
}

\author{
Robert Loce \\ Xerox Research Center Webster \\ 800 Phillips Road \\ Webster, New York 14580 \\ Eli Saber \\ Rochester Institute of Technology \\ 79 Lomb Memorial Drive \\ Rochester, New York 14623
}

The present special section on Video Surveillance and Transportation Imaging was motivated by ubiquity of video surveillance in our everyday environment and transportation venues. With the advent of low-cost/high-performance video sensors, imaging platforms, and computational equipment, it has become increasingly possible to process video streams in real-time on affordable cloud computational servers, desktop systems, and various hand-held mobile devices. Major applications of these technologies span the spectrum from surveillance, transportation, remote sensing, social media, sports, retail, and biomedical to name a few.

Only recently has the potential for these technologies begun to be realized in various commercial, government and consumer-based applications. For instance, automated video understanding can enhance surveillance/monitoring systems beyond what is possible for human operators alone. These systems are being developed to maintain long-term surveillance on large numbers of video streams for various applications with minimal or no manual intervention. In addition, automated systems can coordinate multiple cameras and provide "synopsis" views of activities that can be used to predict/ analyze potential events pre- or postmortem.

On the other hand, transportation systems, the life blood of our economic and social lives, are far from their ideal state. Vehicle accidents are the number one cause of death in the US for ages 4 to 34, and are expected to surpass disease as a cause of death for all ages worldwide by 2020. By some estimates, half the fuel consumed in San Francisco is consumed while searching for parking spaces. Vehicular congestion is a leading cause of lost productivity, and fuel efficiency considerations have traditionally received very limited attention in the design of present-day systems.

We are very pleased to say that the call for papers for this special section on Video Surveillance and Transportation Imaging generated an overwhelming response. From numerous submitted papers, the reviewers have selected the 22 that you will find in this issue. Many transportation applications are presented, such as vehicle classification, surveillance of vehicles, traffic analysis, video-based parking management, driver analysis and assistance, tire imaging, traffic sign recognition, and license plate imaging. A paper on port surveillance is included, and a survey of roadway transportation applications is published here. Several papers are included that address video networking issues. Multipurpose surveillance technologies are represented here, such as scene understanding and tracking. Several papers address surveillance of humans.

We thank the authors for their submissions, and their responses to the detailed reviewers' comments. A great deal of thanks goes to the reviewers. Without an extensive collection of highly competent reviewers, a special section of this size and quality would not be possible. And of course, we thank the staff at JEI for their rapid follow-through on the numerous tasks, the reminders, and the pleasant interactions. 